\title{
Abrupt change in hybridization gap at the valence transition of $\mathrm{YbInCu}_{4}$
}

\author{
Hiroaki Anzai $\odot,{ }^{1, *}$ Suzuna Ishihara, ${ }^{1}$ Kojiro Mimura $\odot,{ }^{1}$ Hitoshi Sato, ${ }^{2}$ Masashi Arita, ${ }^{2}$ Tao Zhuang, ${ }^{3}$ and Koichi Hiraoka ${ }^{3}$ \\ ${ }^{1}$ Graduate School of Engineering, Osaka Prefecture University, Sakai 599-8531, Japan \\ ${ }^{2}$ Hiroshima Synchrotron Radiation Center, Hiroshima University, Higashi-Hiroshima 739-0046, Japan \\ ${ }^{3}$ Graduate School of Science and Engineering, Ehime University, Matsuyama 790-8577, Japan
}

(Received 22 April 2020; accepted 19 August 2020; published 14 September 2020)

\begin{abstract}
The valence transition of $4 f$ electron states in rare-earth compounds is accompanied by a discontinuous change in the Kondo temperature, an energy scale tied to the interaction of the $4 f$ states with the conduction-band states ( $c$ - $f$ hybridization). The $c-f$ hybridization causes the formation of heavy quasiparticle bands and produces a hybridization gap. Whether the hybridization gap develops at the valence-transition temperature $T_{\mathrm{V}}$ is one of the key questions in the $4 f$-electron systems. In our angle-resolved photoemission spectroscopy performed using synchrotron radiation, we observed such a hybridization gap of the valence-transition compound $\mathrm{YbInCu}_{4}$. We found that the gap increases below $T_{\mathrm{V}}$ and has a momentum dependence. Notably, the gap enhancement occurs along with a substantial energy shift of the conduction bands. We suggest that the $c$ - $f$ hybridization and the enhancement of the density of states at $E_{\mathrm{F}}$ should be incorporated in the scenarios of the valence transition in $\mathrm{YbInCu}_{4}$.
\end{abstract}

DOI: 10.1103/PhysRevResearch.2.033408

\section{INTRODUCTION}

The mixed-valence behavior is one of the most intriguing physical phenomena in $4 f$-electron systems. For the Ce- and Yb-based compounds, adjacent $4 f$ configurations are nearly degenerate in energy, and consequently a mixing between the different $4 f$ electron states occurs by small changes in external parameters of temperature, pressure, and magnetic field $[1,2]$. This valence mixing is closely related to the interaction of the localized $4 f$ states with the itinerant conduction-band states ( $c$ - $f$ hybridization) $[3,4]$. Their mutual relationship and strength of the electron interaction are fundamental to the appearance of unusual physical properties of the Ruderman-Kittel-Kasuya-Yosida interaction and Kondo effect [3]. According to the periodic Anderson model, the $c-f$ hybridization gives rise to the formation of heavy quasiparticle bands and produces an energy gap (hybridization gap) at the crossing point of the $4 f$ states and the conduction bands $[5,6]$. Hence, studies in terms of the hybridization gap provide important clues to the driving force for the mixed-valence behavior.

Among the mixed-valence systems, the first-order valence transition at $T_{\mathrm{V}}=42 \mathrm{~K}$ in $\mathrm{YbInCu}_{4}$ has attracted significant attention for a long time. It has been reported from Mössbauer measurements that the $\mathrm{Yb}$ valence changes from $z \simeq 2.9$ in the high-temperature phase to $z \simeq 2.8$ in the low-temperature phase [7]. The valence transition at $T_{\mathrm{V}}$ is accompanied by a

\footnotetext{
*anzai@pe.osakafu-u.ac.jp
}

Published by the American Physical Society under the terms of the Creative Commons Attribution 4.0 International license. Further distribution of this work must maintain attribution to the author(s) and the published article's title, journal citation, and DOI. discontinuous change in physical properties of magnetic susceptibility and electrical resistivity [7-9]. A notable feature is that the Kondo temperature $T_{\mathrm{K}}$, which is a measure of the hybridization strength, varies from $\sim 25 \mathrm{~K}\left(T>T_{\mathrm{V}}\right)$ to $\sim 400 \mathrm{~K}$ $\left(T<T_{\mathrm{V}}\right.$ ) [9]. A key question is whether the magnitude of the hybridization gap in the band dispersions shows a temperature dependence across $T_{\mathrm{V}}$. To understand the mechanism of the valence transition in $\mathrm{YbInCu}_{4}$, we must detect the fingerprints of the $c$ - $f$ hybridization.

Angle-resolved photoemission spectroscopy (ARPES) is a direct technique to measure the electronic excitation as a function of energy and momentum. The momentum dependence of $4 f$ states near the Fermi level $E_{\mathrm{F}}$ has been attributed to their hybridization with valence bands [10-12]. From the recent ARPES studies of $\mathrm{YbInCu}_{4}$, it was revealed that the $\mathrm{Yb} 4 f$ derived band exhibits a momentum-dependent behavior [13]. The evolution of this band in the momentum space and with varying temperatures is still unclear. An extensive dependence study is required to clarify the driving factor involved in the valence transition in $\mathrm{YbInCu}_{4}$.

Here we report on ARPES studies of a typical valencetransition compound $\mathrm{YbInCu}_{4}$. The $4 f$-derived band at $|\omega| \simeq$ $0.13 \mathrm{eV}$ is observed in a wide momentum region and temperature range from well below to well above $T_{\mathrm{V}}$. This band shows a strong momentum-dependent evolution in the twodimensional Brillouin zone of the projected (111) surface. From the calculation using the periodic Anderson model, we demonstrate an enhancement in the hybridization gap below $T_{\mathrm{V}}$, along with an energy shift of the conduction band. We propose the possible scenarios for the valence transition in $\mathrm{YbInCu}_{4}$.

\section{EXPERIMENTAL DETAILS}

High-quality $\mathrm{YbInCu}_{4}$ single crystals were synthesized using the flux method [14,15]. Angle-resolved photoemission 


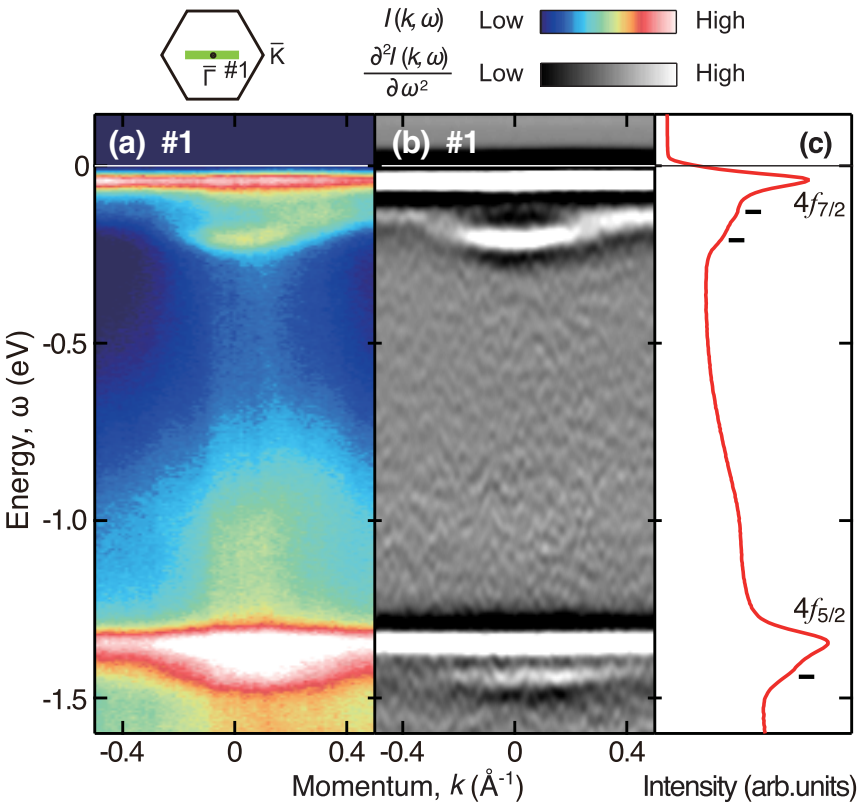

FIG. 1. Angle-resolved photoemission spectroscopy spectrum of $\mathrm{YbInCu}_{4}$ at $T=10 \mathrm{~K}$ in the low-temperature phase. (a) ARPES intensity plot $I(k, \omega)$ taken along the $\bar{\Gamma}-\overline{\mathrm{K}}$ direction (cut 1) of the surface Brillouin zone, as marked by a green line above the image. (b) Second energy derivatives of the ARPES spectrum, $\partial^{2} I(k, \omega) / \partial \omega^{2}$, for the data in (a). (c) Momentum-integrated spectrum of the data in (a). The vertical bars indicate the characteristic energy states.

spectroscopy experiments were performed at BL-9A of the Hiroshima Synchrotron Radiation Center (HSRC). The total energy resolution was set to $17 \mathrm{meV}$. The samples were cleaved in situ along the (111) crystal plane and kept under an ultrahigh vacuum $\left(3.8 \times 10^{-9} \mathrm{~Pa}\right)$ during the measurements. All the data were taken using circularly polarized $h v=24 \mathrm{eV}$ photons. It is known that $\mathrm{YbInCu}_{4}$ exhibits a structural change from the cubic structure in the high-temperature phase to the tetragonal structure in the low-temperature phase. The change in the ratio $c / a$ of the lattice constants is extremely small $(c / a=1.0000004$ at $30 \mathrm{~K})$ [16]. For simplicity, we hereafter use the same surface Brillouin zone with hexagonal shape for both the phases in accordance with the arrangement of $\mathrm{Yb}$ atoms on the (111) plane.

\section{RESULTS AND DISCUSSION}

Figure 1(a) shows the ARPES intensity plot $I(k, \omega)$ along the $\bar{\Gamma}-\bar{K}$ direction (cut 1) of the surface projected Brillouin zone. The data were taken at $T=10 \mathrm{~K}$ in the low-temperature phase. For a better view of the band dispersions, we plot the corresponding second derivative of the ARPES intensity with respect to the energy, $\partial^{2} I(k, \omega) / \partial \omega^{2}$, in Fig. 1(b). Two intense flat bands are clearly seen at $|\omega| \simeq 0.05$ and $1.35 \mathrm{eV}$, which can be assigned to the spin-orbit split $\mathrm{Yb}^{2+} 4 f_{7 / 2}$ and $\mathrm{Yb}^{2+}$ $4 f_{5 / 2}$ states, respectively [17-20]. The momentum-integrated photoemission spectrum is depicted in Fig. 1(c). A broad hump at approximately $|\omega| \simeq 1.0 \mathrm{eV}$ is derived from the contribution of the $\mathrm{Yb}^{2+}$ states at the topmost surface layer [17].
Its weak intensity is sufficient to justify the cleanliness and homogeneity of the sample surface in this study.

In Figs. 1(a) and 1(b), two additional bands can be identified at the high-energy side of the spin-orbit split $\mathrm{Yb} 4 f$ states. At $|\omega| \simeq 1.44 \mathrm{eV}$, the band exhibits a dispersionless feature, which is reflected by the shoulder near the $4 f_{5 / 2}$ state in Fig. 1(c). At $|\omega| \simeq 0.13 \mathrm{eV}$, the other band shows appreciable momentum dependence: The band is flattened at $|k|>0.4 \AA^{-1}$ and curved around the $\bar{\Gamma}$ point. This feature is demonstrated by the double peaks near the $4 f_{7 / 2}$ state in Fig. 1(c). The splitting width and relative intensity of the flat band with respect to the $4 f_{5 / 2}$ peak coincide well with those of the spin-orbit partner of the $4 f_{7 / 2}$ peak. Therefore, the two bands at 0.13 and $1.44 \mathrm{eV}$ originate from the $\mathrm{Yb} 4 f$ states.

The magnified view of the spectrum in Figs. 1(a) and 1(b) are depicted in Figs. 2(a) and 2(c), respectively. We found the splitting of the $0.13-\mathrm{eV}$ band: One branch is shifted downward in energy to $|\omega| \simeq 0.21 \mathrm{eV}$ and the other is shifted upward in energy to $|\omega| \simeq 0.12 \mathrm{eV}$, as seen from the peaks in the energy distribution curves (EDCs) of Fig. 2(e). At momentum cut 2 in the region intermediate to the zone boundary, the momentumdependent behavior of the $0.13-\mathrm{eV}$ band significantly changes. The band exhibits parabolic dispersion at $|k|<0.15 \AA^{-1}$, as shown in Figs. 2(b), 2(d), and 2(f). Such a dentlike shape in $4 f$-derived bands has been observed in $\mathrm{Yb}$ compounds and is assigned to hybridization phenomena $[10,11]$. This similarity implies the hybridization between the $4 f$-derived states and conduction bands in $\mathrm{YbInCu}_{4}$.

For a quantitative analysis, we now compare our observations to the periodic Anderson model $[6,10]$. In this model, the two reconstructed bands $\left(E_{k}^{+}\right.$and $\left.E_{k}^{-}\right)$due to the $c-f$ hybridization are described by form

$$
E_{k}^{ \pm}=\frac{1}{2}\left\{\varepsilon_{f}+\varepsilon_{c} \pm \sqrt{\left(\varepsilon_{f}-\varepsilon_{c}\right)^{2}+4 V_{k}^{2}}\right\}
$$

where $\varepsilon_{c}$ and $\varepsilon_{f}$ denote the conduction-band and $f$-band energies, respectively. The hybridization energy $2 V_{k}$ represents the strength of orbital hybridization and it is reflected by the size of the energy gap at the crossing point of $\varepsilon_{c}$ and $\varepsilon_{f}$.

Figures 3(a) and 3(b) display the calculated spectral function $A(k, \omega)$ with hybridization energies of $2 V_{k}=0.04$ and $0.12 \mathrm{eV}$, respectively. We note that the spectral weight of the spin-orbit split $4 f_{7 / 2}$ state at $|\omega| \simeq 0.05 \mathrm{eV}$ is not included in this calculation for simplicity. The peak width of the simulated spectrum is set to be comparable to that of the EDCs in Figs. 2(e) and 2(f). Our simulations are in excellent agreement with the shape of the $0.13-\mathrm{eV}$ band shown in Figs. 2(a)-2(d). A threefold enhancement of $2 V_{k}$ in the intermediate region indicates the effect of momentum-dependent hybridization in $\mathrm{YbInCu}_{4}$. The parameter set for the conduction band approximately reproduces the shape of the dispersions obtained from the band-structure calculation including the effect of the on-site $4 f$ Coulomb interaction $[21,22]$.

The temperature dependence of $\partial^{2} I(k, \omega) / \partial \omega^{2}$ along momentum cuts 1 and 2 is depicted in Figs. 4(a 1)-4(a 3) and 4(b 1)-4(b 3), respectively. The band remains at $|\omega| \simeq$ $0.13 \mathrm{eV}$ far above $T_{\mathrm{V}}=42 \mathrm{~K}$. We observed an abrupt change in the energy of the band bottom of the lower branch at $T_{\mathrm{V}}$. As seen from the peak positions (vertical bars) of the EDCs at $k=0 \AA^{-1}$ in Figs. 4(c) and 4(d), the energy of the peak 

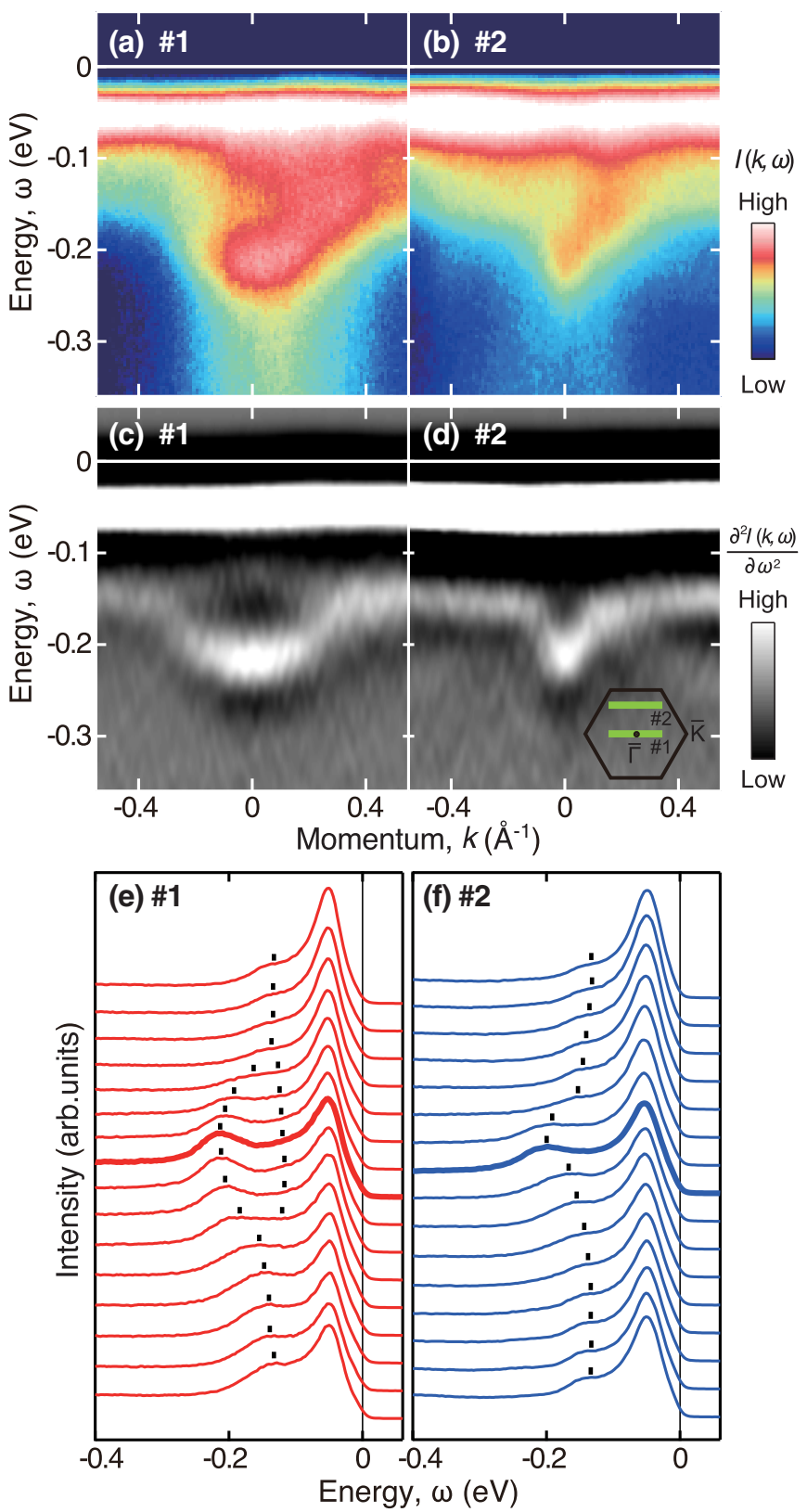

FIG. 2. Momentum dependence of the ARPES spectrum in the low-temperature phase. The ARPES intensity plot $I(k, \omega)$ is taken along momentum cuts (a) 1 and (b) 2, as marked in the inset of (d). (c) and (d) Second energy derivatives of the ARPES spectrum, $\partial^{2} I(k, \omega) / \partial \omega^{2}$, for the data in (a) and (b), respectively. (e) and (f) Energy distribution curves for the data in (a) and (b), respectively. The vertical bars represent the peak positions of EDCs, except for the peak of the $\mathrm{Yb}^{2+} 4 f_{7 / 2}$ state at $|\omega| \simeq 0.05 \mathrm{eV}$.

exhibits a clear steplike increase near $T_{\mathrm{V}}$ and tends to saturate above $T_{\mathrm{V}}$. The peak positions are plotted in Fig. 4(e) as a function of temperature. The total shifts in the energy are estimated to be $\Delta_{1} \simeq 0.03$ and $\Delta_{2} \simeq 0.04 \mathrm{eV}$ in cuts 1 and 2 , respectively. The steplike variation in the physical properties at $T_{\mathrm{V}}$ is a typical behavior of the valence-transition system [7-9]. Therefore, the observed bands are associated with the valence-transition phenomenon in $\mathrm{YbInCu}_{4}$.

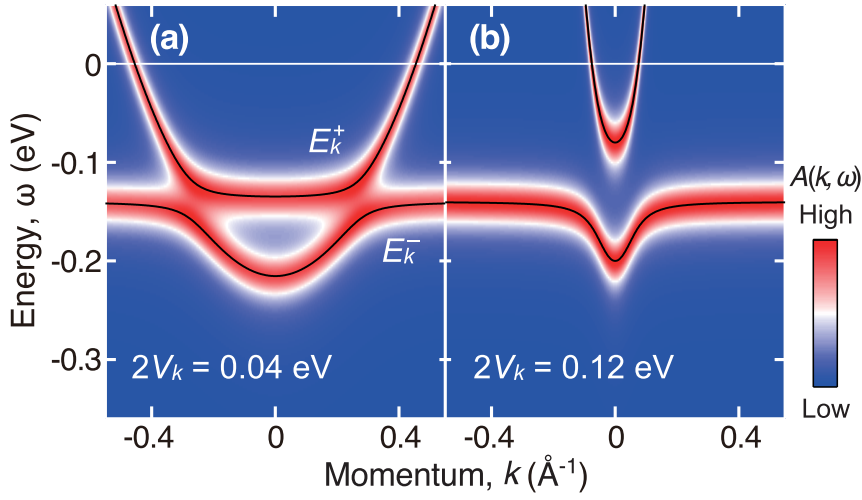

FIG. 3. (a) and (b) Spectral function $A(k, \omega)$ calculated using the periodic Anderson model in Eq. (1). The solid curves represent the upper, $E_{k}^{+}$, and lower, $E_{k}^{-}$, branches. The hybridization energies are (a) $2 V_{k}=0.04 \mathrm{eV}$ and (b) $2 V_{k}=0.12 \mathrm{eV}$. The conduction band is assumed to be an electronlike parabolic dispersion $a+b \omega^{2}$. The parameters are (a) $a=-0.21$ and $b=1$ and (b) $a=-0.14$ and $b=$ 20. The linewidth broadening of the spectrum is set to be comparable to the peak width of the EDCs in Figs. 2(e) and 2(f). The energy of the spin-orbit $4 f_{7 / 2}$ band is excluded in this calculation for simplicity.

We here examine the possible evolution of the hybridized bands. In the spectrum along momentum cut 1, the energy shift of the band bottom due to the effect of the $c-f$ hybridization is negligible, because the hybridization gap opens at $|k| \simeq 0.27 \AA^{-1}$, where the conduction band crosses the $4 f$-derived bands. As discussed in the Appendix, the band bottom energy remains almost unchanged irrespective of the opening and closing of the hybridization gap. We therefore consider that the energy shift in cut 1 results from the energy shift of the electronlike conduction band at $T_{\mathrm{V}}$. This band shift enhances the density of state at $E_{\mathrm{F}}$, leading to the reduction of the electrical resistivity below $T_{\mathrm{V}}[8,9]$.

For the spectrum along cut 2, a large hybridization gap of $2 V_{k}=0.12 \mathrm{eV}$ is formed below $T_{\mathrm{V}}$, as shown in Fig. 3(b). Considering a rigid-bandlike shift, the conduction band shifts by the same amount of $0.03 \mathrm{eV}$ that is observed in cut 1 . However, the peak shift of the EDCs in cut 2 is $0.04 \mathrm{eV}$, as shown in Figs. 4(d) and 4(e). Therefore, a change in the hybridization energy is required to explain our experimental data.

Figures 4(f) and 4(g) show the calculated spectral function with hybridization energies of $2 V_{k}=0.12 \mathrm{eV}\left(T<T_{\mathrm{V}}\right)$ and $2 V_{k}=0.07 \mathrm{eV}\left(T>T_{\mathrm{V}}\right)$, respectively. We note that the former is the same datum presented in Fig. 3(b). The conduction band with a parabolic dispersion in Fig. $4(\mathrm{~g})$ is shifted toward $E_{\mathrm{F}}$ in accordance with the rigid-bandlike shift of $0.03 \mathrm{eV}$. We have obtained a consistency that a $0.05-\mathrm{eV}$ change in the hybridization energy reproduces the momentum- and temperature-dependent behavior of the $0.13-\mathrm{eV}$ band shown in Figs. 4(b 1)-4(b 3). These data provide the evidence that the enhancement of the hybridization gap, as well as that of the density of states at $E_{\mathrm{F}}$, is crucial for the valence transition of $\mathrm{YbInCu}_{4}$. This concept is supported by the recent resonant inelastic $\mathrm{x}$-ray-scattering and ultrafast pump-probe measurements [23,24]. 


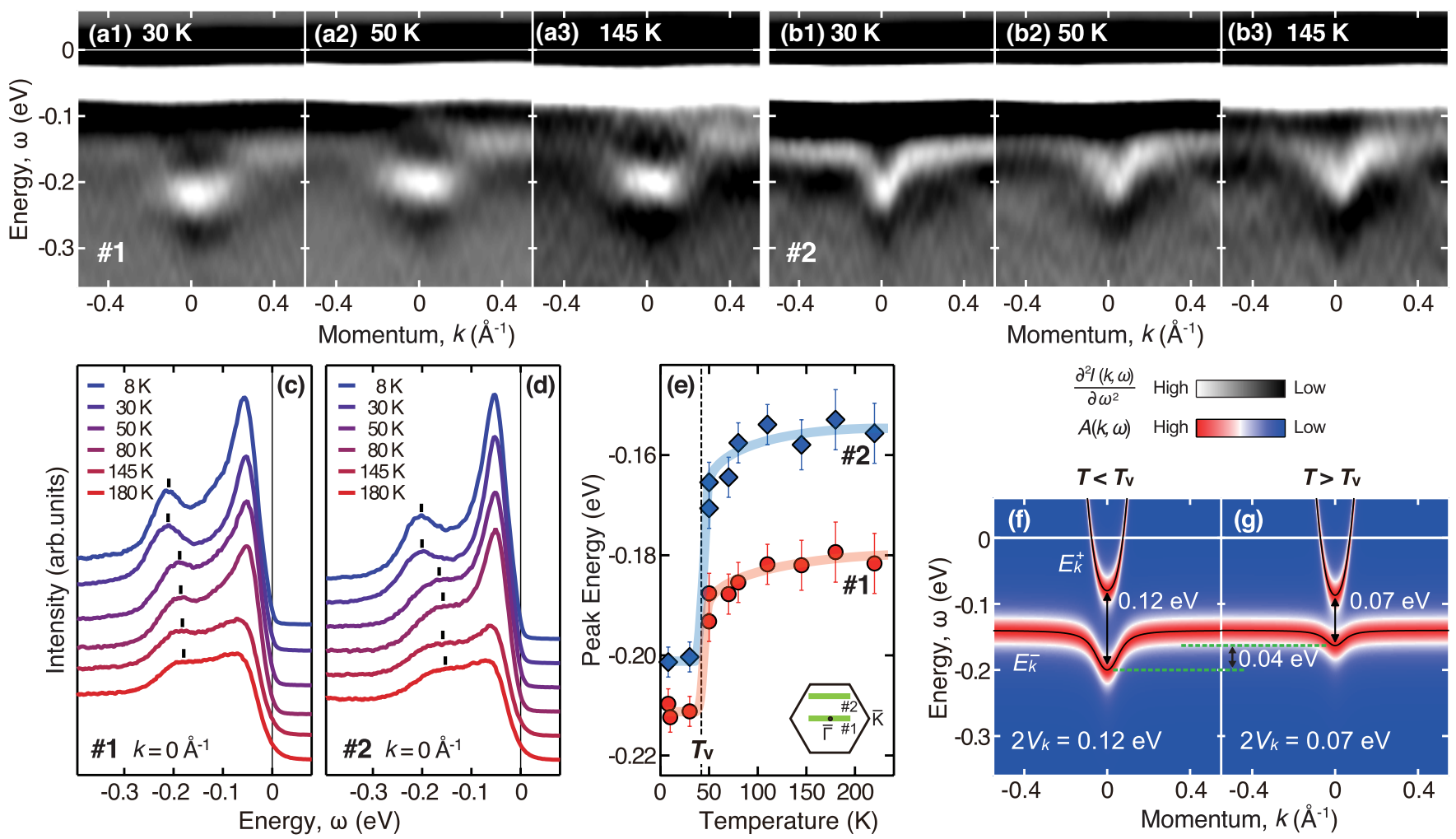

FIG. 4. Temperature evolution of the electronic band structure. (a) Second energy derivatives of the ARPES intensity, $\partial^{2} I(k, \omega) / \partial \omega^{2}$, at various temperatures measured along the $\bar{\Gamma}-\bar{K}$ direction: (a 1) $30 \mathrm{~K}$, (a 2 ) $50 \mathrm{~K}$, and (a 3) $145 \mathrm{~K}$. (b) Same data set as in (a), but along momentum cut 2, as marked in the inset of (e): (b 1) $30 \mathrm{~K}$, (b 2) $50 \mathrm{~K}$, and (b 3 ) $145 \mathrm{~K}$. The temperature dependence of the EDCs at $k=0 \AA^{-1}$ were taken from momentum cuts (c) 1 and (d) 2 . The vertical bars represent the peak positions of the lower branch of the hybridized bands. (e) Peak energies shown in (c) and (d) as a function of temperature. The error bars stem from the natural broadness of the EDC peaks. The thick curves are guides for the eye. (f) Same data as in Fig. 3(b) with the hybridization energy $2 V_{k}=0.12 \mathrm{eV}$. (g) Calculated spectral function $A(k, \omega)$ with $2 V_{k}=0.07 \mathrm{eV}$. The band parameters for the electronlike conduction band $a+b \omega^{2}$ are set to $a=-0.11$ and $b=20$. The solid curves represent the upper, $E_{k}^{+}$, and lower, $E_{k}^{-}$, branches in Eq. (1).

We next discuss a possible origin of the $0.13-\mathrm{eV}$ band. In a previous ARPES study of $\mathrm{YbRh}_{2} \mathrm{Si}_{2}$, the crystal-field splitting of $4 f$ states has been observed near $E_{\mathrm{F}}$ [11]. For the case of $\mathrm{YbInCu}_{4}$, the eightfold degenerate Hund's rule ground state of $\mathrm{Yb}^{3+}$ with $J=7 / 2$ splits into two doublets and one quartet. The overall splitting energy is approximately $8 \mathrm{meV}[25,26]$. This value is 10 times smaller than the energy difference between the band at $|\omega| \simeq 0.13 \mathrm{eV}$ and the $4 f_{7 / 2}$ state at $|\omega| \simeq 0.05 \mathrm{eV}$. Therefore, the $0.13-\mathrm{eV}$ band cannot be assigned to the crystal-field-split $4 f$ states.

It is conceivable that the $0.13-\mathrm{eV}$ band originates from the $\mathrm{Yb} 4 f$ states in the subsurface region that intervenes between the surface and bulk regions of $\mathrm{YbInCu}_{4}$ [18-20]. The electronic state of the subsurface region exhibits characteristics intermediate between those at the surface and those in the bulk [20]. The band is indeed observed between the surfacederived state at $|\omega| \simeq 1.0 \mathrm{eV}$ and the bulk-derived $4 f_{7 / 2}$ state at $|\omega| \simeq 0.05 \mathrm{eV}$, as shown in Fig. 1(c). On this basis, we infer that the $0.13-\mathrm{eV}$ band is derived from the $\mathrm{Yb} 4 f$ states in the subsurface region of the crystal.

The contribution of the In $5 p$ states to the valence transition has been identified by comparing the spectra of $\mathrm{YbCdCu}_{4}$ and $\mathrm{YbSnCu}_{4}$ [27-30]. Furthermore, the resonant inelastic X-rayscattering measurements has revealed an energy shift of the itinerant $\mathrm{Yb} 5 d$ states and an increase in the density of states at $E_{\mathrm{F}}$ below $T_{\mathrm{V}}$ [23]. Therefore, the In $5 p$ and $\mathrm{Yb} 5 d$ states are responsible for the hybridization with the $\mathrm{Yb} 4 f$ states [4]. We emphasize that the steplike variation of the hybridized band results from the energy shift of the bulk conduction band. This energy shift may be attributed to the structural phase transition at $T_{\mathrm{V}}[16]$.

Regarding the spin-orbit split $4 f_{7 / 2}$ state at $|\omega| \simeq 0.05 \mathrm{eV}$, a gaplike feature has not been observed thus far. The intense intensity of the $4 f_{7 / 2}$ state eliminates the fine feature of the band dispersion near $E_{\mathrm{F}}$. However, it is reasonable that the $4 f_{7 / 2}$ state also hybridizes with the conduction band, as is the case for $\mathrm{YbAl}_{2}$ [31]. Further experiments are required to clarify the landscape of conduction bands such as Fermi surfaces. Irrespective of this question, our results provide evidence for the abrupt change in the hybridization gap at $T_{\mathrm{V}}$, highlighting the hybridization of the $4 f$-derived band with the bulk conduction band in $\mathrm{YbInCu}_{4}$.

\section{CONCLUSION}

In conclusion, our APRES measurements on $\mathrm{YbInCu}_{4}$ have revealed a strong momentum-dependent behavior of the $4 f$-derived band at $|\omega| \simeq 0.13 \mathrm{eV}$. A threefold increase in the 

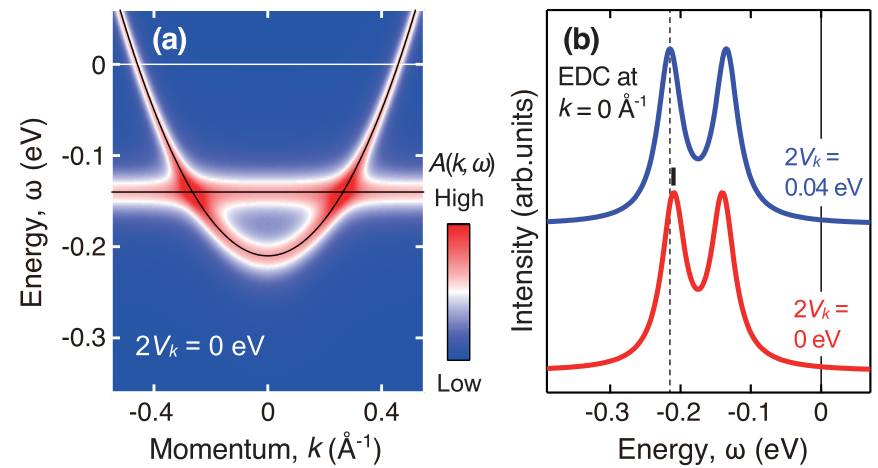

FIG. 5. (a) Spectral function $A(k, \omega)$ calculated by the periodic Anderson model. The hybridization energy of Eq. (1) is set to $2 V_{k}=0 \mathrm{eV}$. (b) EDCs at $k=0 \AA^{-1}$ taken from (a) and Fig. 3(a). The red and blue lines are EDCs without and with the hybridization energy, respectively. The vertical thick and dashed lines denote peak positions of the EDCs with $2 V_{k}=0$ and $0.04 \mathrm{eV}$, respectively.

hybridization energy in the momentum space is demonstrated by the comparison of the experimental observations with the periodic Anderson model. Notably, the band bottom of the lower branch exhibits the steplike energy shift at $T_{\mathrm{V}}$. Such a nonlinear evolution is a typical behavior of valence-transition phenomena. The temperature dependence of the band dispersions can be explained by the fact that the hybridization energy increases by $0.05 \mathrm{eV}$ along with a substantial energy shift of the electronlike conduction bands below $T_{\mathrm{V}}$. We suggest that the $c$ - $f$ hybridization and the enhancement of the density of state at $E_{\mathrm{F}}$ are essential parts of the mechanism for the valence transition of $\mathrm{YbInCu}_{4}$.

\section{ACKNOWLEDGMENTS}

We thank K. Shimada for valuable discussions. This work was supported by the Specific Support Project of Osaka Prefecture University. The ARPES experiments were performed under the approval of HSRC (Proposals No. 16AG032 and No. 16BG055)

\section{APPENDIX}

One of our important findings is the observation of a shift of the conduction band at the valence-transition temperature $T_{\mathrm{V}}$. This behavior can be seen in the spectrum of $\partial^{2} I(k, \omega) / \partial \omega^{2}$ along the $\bar{\Gamma}-\bar{K}$ direction (cut 1$)$ of Figs. 4(a 1$)$ and 4(a 2$)$ and in the EDCs of Fig. 4(c). However, one might argue that this energy shift is alternatively interpreted as a change in the strength of $c-f$ hybridization. In this Appendix we show that the band bottom energy along the cut 1 direction remains almost unchanged regardless of the opening and closing of the hybridization gap.

Figure 5(a) shows the calculated image plotted without the hybridization energy $\left(2 V_{k}=0 \mathrm{eV}\right)$ of Eq. (1). A flat band at $|\omega|=0.13 \mathrm{eV}$ and a parabolic band of $-0.21+\omega^{2}$ intersect at $|k| \simeq 0.27 \AA^{-1}$. We plotted the EDC at $k=0 \AA^{-1}$ in Fig. 5(b), together with the EDC $\left(2 V_{k}=0.04 \mathrm{eV}\right)$ of Fig. 3(a). The energy of the band bottom remains approximately at the same energy. This is inconsistent with the experimental observation shown in Figs. 4(a 1), 4(a 2), 4(c), and 4(e). Therefore, we consider the energy shift of the conduction band at $T_{\mathrm{V}}$ to be a reasonable explanation for the temperature dependence of ARPES spectra.
[1] C. M. Varma, Mixed-valence compounds, Rev. Mod. Phys. 48, 219 (1976)

[2] B. Johansson, Energy position of $4 f$ levels in rare-earth metals, Phys. Rev. B 20, 1315 (1979).

[3] S. Doniach, The Kondo lattice and weak antiferromagnetism, Physica B+C 91, 231 (1977).

[4] E. Figueroa, J. M. Lawrence, J. L. Sarrao, Z. Fisk, M. F. Hundley, and J. D. Thompson, Hall effect in $\mathrm{YbXCu}_{4}$ and the role of carrier density in the $\mathrm{YbInCu}_{4}$ valence transition, Solid State Commun. 106, 347 (1998).

[5] A. C. Hewson, The Kondo Problem to Heavy Fermions (Cambridge University Press, Cambridge, 1993).

[6] A. N. Tahvildar-Zadeh, M. Jarrell, and J. K. Freericks, LowTemperature Coherence in the Periodic Anderson Model: Predictions for Photoemission of Heavy Fermions, Phys. Rev. Lett. 80, 5168 (1998).

[7] I. Felner, I. Nowik, D. Vaknin, U. Potzel, J. Moser, G. M. Kalvius, G. Wortmann, G. Schmiester, G. Hilscher, E. Gratz, C. Schmitzer, N. Pillmayr, K. G. Prasad, H. de Waard, and H. Pinto, Ytterbium valence phase transition in $\mathrm{Yb}_{x} \mathrm{In}_{1-x} \mathrm{Cu}_{2}$, Phys. Rev. B 35, 6956 (1987).

[8] B. Kindler, D. Finsterbusch, R. Graf, F. Ritter, W. Assmus, and B. Luthi, Mixed-valence transition in $\mathrm{YbInCu}_{4}$, Phys. Rev. B 50, 704 (1994).
[9] J. L. Sarrao, C. D. Immer, C. L. Benton, and Z. Fisk, Evolution from first-order valence transition to heavy-fermion behavior in $\mathrm{YbIn}_{1-x} \mathrm{Ag}_{x} \mathrm{Cu}_{4}$, Phys. Rev. B 54, 12207 (1996).

[10] S. Danzenbächer, Y. Kucherenko, D. V. Vyalikh, M. Holder, C. Laubschat, A. N. Yaresko, C. Krellner, Z. Hossain, C. Geibel, X. J. Zhou, W. L. Yang, N. Mannella, Z. Hussain, Z.-X. Shen, M. Shi, L. Patthey, and S. L. Molodtsov, Momentum dependence of $4 f$ hybridization in heavy-fermion compounds: Angle-resolved photoemission study of $\mathrm{YbIr}_{2} \mathrm{Si}_{2}$ and $\mathrm{YbRh}_{2} \mathrm{Si}_{2}$, Phys. Rev. B 75, 045109 (2007).

[11] D. V. Vyalikh, S. Danzenbächer, Y. Kucherenko, K. Kummer, C. Krellner, C. Geibel, M. G. Holder, T. K. Kim, C. Laubschat, M. Shi, L. Patthey, R. Follath, and S. L. Molodtsov, $k$ Dependence of the Crystal-Field Splittings of $4 f$ States in Rare-Earth Systems, Phys. Rev. Lett. 105, 237601 (2010).

[12] H. Anzai, K. Ichiki, E. F. Schwier, H. Iwasawa, M. Arita, H. Sato, K. Shimada, H. Namatame, M. Taniguchi, A. Mitsuda, H. Wada, and K. Mimura, Observation of the $c$ - $f$ hybridization effect in valence-transition system EuPtP, Phys. Status Solidi C 14, 1600185 (2017).

[13] S. Ishihara, K. Ichiki, K. Abe, T. Matsumoto, K. Mimura, H. Sato, M. Arita, E. F. Schwier, H. Iwasawa, K. Shimada, H. Namatame, M. Taniguchi, T. Zhuang, K. Hiraoka, and H. Anzai, The $c$ - $f$ hybridization effect in the subsurface region 
of $\mathrm{YbInCu}_{4}$, J. Electron Spectrosc. Relat. Phenom. 220, 66 (2017).

[14] K. Hiraoka, K. Murakami, S. Tomiyoshi, T. Hihara, T. Shinohara, and K. Kojima, ${ }^{63} \mathrm{Cu}$ NQR and ${ }^{113} \mathrm{Cd}$ NMR studies of $\mathrm{YbMCu}_{4}(\mathrm{M}=\mathrm{In}, \mathrm{Cd})$, Physica B (Amsterdam) 281-282, 173 (2000).

[15] C. Moriyoshi, S. Shimomura, K. Itoh, K. Kojima, and K. Hiraoka, Crystal structure and valence transition temperature of $\mathrm{YbInCu}_{4}$ single crystals, J. Magn. Magn. Mater. 260, 206 (2003).

[16] S. Tsutsui, K. Sugimoto, R. Tsunoda, Y. Hirose, T. Mito, R. Settai, and M. Mizumaki, First-order structural change accompanied by $\mathrm{Yb}$ valence transition in $\mathrm{YbInCu}_{4}$, J. Phys. Soc. Jpn. 85, 063602 (2016).

[17] F. Reinert, R. Claessen, G. Nicolay, D. Ehm, S. Hüfner, W. P. Ellis, G.-H. Gweon, J. W. Allen, B. Kindler, and W. Assmus, Photoemission experiments on $\mathrm{YbInCu}_{4}$ : Surface effects and temperature dependence, Phys. Rev. B 58, 12808 (1998).

[18] H. Sato, K. Hiraoka, M. Taniguchi, Y. Takeda, M. Arita, K. Shimada, H. Namatame, A. Kimura, K. Kojima, T. Muro, Y. Saitoh, A. Sekiyama and S. Suga, Electronic structure of $\mathrm{YbXCu}_{4}(X=\mathrm{In}, \mathrm{Cd}, \mathrm{Mg})$ investigated by high-resolution photoemission spectroscopy, J. Synchrotron Radiat. 9, 229 (2002).

[19] H. Sato, K. Yoshikawa, K. Hitaoka, M. Arita, K. Fujimoto, K. Kojima, T. Muro, Y. Saitoh, A. Sekiyama, S. Suga, and M. Taniguchi, Soft-x-ray high-resolution photoemission study on the valence transitions in $\mathrm{YbInCu}_{4}$, Phys. Rev. B 69, 165101 (2004).

[20] S. Suga, A. Sekiyama, S. Imada, J. Yamaguchi, A. Shigemoto, A. Irizawa, K. Yoshimura, M. Yabashi, K. Tamasaku, A. Higashiya, and T. Ishikawa, Unraveling genuine first order bulk valence transition and Kondo resonance behaviors in $\mathrm{YbInCu}_{4}$ by high energy photoelectron spectroscopy, J. Phys. Soc. Jpn. 78, 074704 (2009).

[21] K. Takegahara and T. Kasuya, Electronic band structure of $\mathrm{YbInCu}_{4}$ and LuInCu 4 , J. Phys. Soc. Jpn. 59, 3299 (1990).

[22] V. N. Antonov, M. Galli, and F. Marabelli, Electronic structure and optical spectra of $\mathrm{LuInCu}_{4}$ and $\mathrm{YbMCu}_{4}(M=\mathrm{Cu}, \mathrm{Ag}, \mathrm{Au}$, Pd, and In), Phys. Rev. B 62, 1742 (2000).

[23] I. Jarrige, A. Kotani, H. Yamaoka, N. Tsujii, K. Ishii, M. Upton, D. Casa, J. Kim, T. Gog, and J. N. Hancock, Kondo Interactions from Band Reconstruction in $\mathrm{YbInCu}_{4}$, Phys. Rev. Lett. 114, 126401 (2015).

[24] M. Y. Zhang, R. Y. Chen, T. Dong, and N. L. Wang, Dramatic change of photoexcited quasiparticle relaxation dynamics across $\mathrm{Yb}$ valence state transition in $\mathrm{YbInCu}_{4}$, Phys. Rev. B 95, 165104 (2017).

[25] G. Polatsek and P. Bonville, Interpretation of neutron magnetic scattering in the Kondo lattices $\mathrm{YbPd}_{2} \mathrm{Si}_{2}$ and $\mathrm{YbAgCu}_{4}$, Z. Phys. B 88, 189 (1992).

[26] T. Willers, J. C. Cezar, N. B. Brookes, Z. Hu, F. Strigari, P. Körner, N. Hollmann, D. Schmitz, A. Bianchi, Z. Fisk, A. Tanaka, L. H. Tjeng, and A. Severing, Magnetic Field Induced Orbital Polarization in Cubic $\mathrm{YbInNi}_{4}$ : Determining the Quartet Ground State Using X-Ray Linear Dichroism, Phys. Rev. Lett. 107, 236402 (2011).

[27] K. Yoshikawa, H. Sato, M. Arita, Y. Takeda, K. Hiraoka, K. Kojima, K. Tsuji, H. Namatame, and M. Taniguchi, Low-energy excited photoemission study of the valence transition of $\mathrm{YbInCu}_{4}$, Phys. Rev. B 72, 165106 (2005).

[28] Y. Utsumi, H. Sato, K. Tobimatsu, H. Maso, K. Hiraoka, K. Kojima, K. Mimura, S. Ueda, Y. Yamashita, H. Yoshikawa, K. Kobayashi, K. Shimada, H. Namatame, and M. Taniguchi, $X$-dependent electronic structure of $\mathrm{YbXCu}_{4} \quad(X=\mathrm{Cd}$, In, Sn) investigated by hard X-ray photoemission spectroscopy, J. Electron Spectrosc. Relat. Phenom. 184, 203 (2011).

[29] H. Anzai, S. Ishihara, H. Shiono, K. Morikawa, T. Iwazumi, H. Sato, T. Zhuang, K. T. Matsumoto, and K. Hiraoka, Mixedvalence state of the rare-earth compounds $\mathrm{YbXCu}_{4}(X=\mathrm{Mg}$, $\mathrm{Cd}, \mathrm{In}$, and Sn): Magnetic susceptibility, $\mathrm{x}$-ray diffraction, and X-ray absorption spectroscopy investigations, Phys. Rev. B 100, 245124 (2019).

[30] H. Anzai, K. Morikawa, H. Shiono, H. Sato, S.-i. Ideta, K. Tanaka, T. Zhuang, K. T. Matsumoto, and K. Hiraoka, Temperature dependence of Kondo resonance in photoemission spectra of the heavy-fermion compounds $\mathrm{YbXCu}_{4}(X=\mathrm{Mg}, \mathrm{Cd}$, and Sn), Phys. Rev. B 101, 235160 (2020).

[31] M. Matsunami, T. Hajiri, H. Miyazaki, M. Kosaka, and S. Kimura, Strongly hybridized electronic structure of $\mathrm{YbAl}_{2}$ : An angle-resolved photoemission study, Phys. Rev. B 87, 165141 (2013). 